\title{
Correlation transfer and diffusion of ultrasound-modulated multiply scattered light
}

\author{
Sava Sakadžić and Lihong V. Wang \\ Optical Imaging Laboratory, Department of Biomedical Engineering, \\ Texas A\&M University, College Station, Texas 77843-3120
}

\begin{abstract}
We develop a temporal correlation transfer equation (CTE) and a temporal correlation diffusion equation (CDE) for ultrasound-modulated multiply scattered light, which can be used to calculate the ultrasound-modulated optical intensity in an optically scattering medium with a nonuniform ultrasound field and a heterogeneous distribution of optical parameters. We present an analytical solution based on the CDE for scattering of the temporal autocorrelation function from a cylinder of ultrasound in an optically scattering slab. The CDE is valid for moderate ultrasound pressures on a scale comparable with the optical transport mean free path, which must be greater than the ultrasound wavelength and smaller than or comparable to the sizes of both ultrasonic and optical inhomogeneities. These equations should be applicable to a wide spectrum of conditions for ultrasoundmodulated optical tomography of soft biological tissues.
\end{abstract}

Keywords: ultrasound-modulated optical tomography, multiply scattered light, correlation transfer equation, diffusion approximation, turbid medium

\section{INTRODUCTION}

The optical properties of tissues in visible and near-infrared regions are related to their molecular structure. Radiation at these wavelengths is nonionizing with potential for the functional imaging and detection of tissue abnormalities. However, due to the diffusion of light, it is difficult to simultaneously achieve both good spatial resolution and good imaging depth with the pure optical imaging modalities, such as optical coherence tomography or diffuse optical tomography. Therefore, much effort has recently been expended to advance soft biological tissue imaging based on ultrasound-modulated multiply scattered light.

Ultrasound-modulated optical tomography (UOT) is a hybrid technique which combines ultrasonic resolution and optical contrast. It is being proposed in order to provide better resolution for the optical imaging of soft biological tissues at imaging depths where light is completely diffused. With this technique, ${ }^{1,2}$ optical radiation, which has high temporal coherence, and focused ultrasound are applied simultaneously to soft biological tissue. The intensity of the ultrasound-modulated optical radiation is related to the optical properties of the tissue in the interaction region of the ultrasonic and electromagnetic waves.

Efficient detection of ultrasound-modulated optical intensity is challenging because of the diffused light propagation and the uncorrelated phases among the optical speckles. At present, the development of effective detection systems is the subject of intense research. ${ }^{3-10}$ Also, the exact nature of the ultrasound-modulation of light in a highly optically scattering medium is still not totally understood due to the complicated light-ultrasound interaction in the presence of optical scatterers. Similarly to dynamic light scattering by scatterers undergoing Brownian motion, ${ }^{11}$ dynamic scattering by optical scatterers oscillating in an ultrasound field causes optical frequency shifts. ${ }^{12,13}$ Optical frequency shifts are also produced due to ultrasound-induced changes in the optical index of refraction. ${ }^{14,15}$ A theoretical model, ${ }^{15}$ which combines both mechanisms of modulation, has been subsequently extended to account for anisotropic optical scattering, ${ }^{16}$ and Brownian motion. ${ }^{16,17}$ Additional extensions of the theoretical model include pulsed ultrasound ${ }^{18}$ and strong correlations between the ultrasoundinduced optical phase increments which exist when the optical transport mean free path $l_{t r}$ is smaller than the ultrasound wavelength $\lambda_{a}{ }^{18}$ A Monte Carlo algorithm has also been developed, ${ }^{19}$ which can be adapted to explore complex geometries.

Existing theoretical models are based on the diffusing-wave spectroscopy (DWS) approach, ${ }^{11,20}$ where the interaction of a plane ultrasound wave with diffused light is considered in an infinite scattering medium. As

Photons Plus Ultrasound: Imaging and Sensing 2006: The 7th Conference on Biomedical
Thermoacoustics, Optoacoustics, and Acousto-optics, edited by A. A. Oraevsky, et. al

Proc. of SPIE Vol. 6086, 60861T (2006) • 1605-7422/06/\$15 • doi: 10.1117/12.645282

Proc. of SPIE Vol. 6086 60861T-1 
a result, analytical solutions are limited to simple geometries where the ultrasound field can be approximated as a plane wave and where the probability density function of the optical path length is analytically known. Consequently, only transmission through, ${ }^{15}, 16,18$ and reflection from, ${ }^{17,18}$ an infinite scattering slab filled with ultrasound have been analytically studied. In practice, however, the ultrasound field is focused and the optical parameters are distributed heterogeneously. Therefore, a more general theoretical model is needed.

In this proceeding, we formally derive a temporal correlation transfer equation (CTE) and a temporal correlation diffusion equation (CDE) for ultrasound-modulated multiply scattered light for isotropic optical scattering and $k_{a} l_{t r} \gg 1$, where $k_{a}=2 \pi / \lambda_{a}$ is the magnitude of the ultrasound wave vector. These equations can be used to obtain both analytical and numerical solutions for the distribution of the modulated light intensity in scattering samples with heterogeneous optical parameters and a nonuniform ultrasound field. In addition, the simple form of the $\mathrm{CDE}$ benefits from all of the mathematical tools available for the diffusion equation. A derivation of a more complex CTE based on the ladder approximation of the Bethe-Sapleter equation will be presented elsewhere. ${ }^{21}$

\section{DEVELOPMENT OF THE CORRELATION TRANSFER EQUATION}

We consider the interaction of ultrasound with monochromatic light that diffuses through the medium with discrete, uncorrelated optical scatterers. The scattering is assumed to be independent, and, for simplicity, we further neglect the polarization of the electrical field. Under the weak scattering approximation that the optical mean free path is much greater than the optical wavelength, the transfer of light can be described by ladder diagrams. ${ }^{22}$ In our case, this also involves calculation of the optical phase increments due to both mechanisms of the ultrasound modulation along the optical paths. The phase increments are generally correlated if they originate at positions separated by less than $l_{t r}$ or $\lambda_{a},{ }^{18}$ which creates difficulties in the derivation of a simple transfer-like equation for the temporal correlation of ultrasound-modulated light. ${ }^{21}$ However, a simple form of CTE can be obtained when $k_{a} l_{t r} \gg 1$. At scales larger than $l_{t r}$, the effect of ultrasound modulation can be calculated by assuming isotropic scattering, where $l_{t r}$ is used instead of the mean-free path. ${ }^{16,18,19}$ The condition $k_{a} l_{t r} \gg 1$ then ensures that the ultrasound-induced optical phase increments associated with the different scattering events are independent. The only correlation between phase increments which then exists is between (1) the phase increment that is due to index of refraction changes along the free path and (2) the phase increments that are due to displacements of these two scatterers along the free path. ${ }^{18}$ This allows for a simple form of the CTE that is valid for optical and ultrasonic inhomogeneities comparable with $l_{t r}$. In soft biological tissues, $l_{t r} \approx 1 \mathrm{~mm}$ for visible and near infrared optical wavelengths, and $k_{a} l_{t r}>10$ for ultrasound frequencies greater than $2.4 \mathrm{MHz}$.

Consider optical scatterers at resting positions $\mathbf{r}_{a}$ and $\mathbf{r}_{b}$, and assume that the ultrasound field can be locally approximated as by $P(\vec{r}, t)=P_{0} \cos \left(\omega_{a} t-\mathbf{k}_{a} \cdot \mathbf{r}+\phi\right)$, where $\mathbf{k}_{a}=k_{a} \hat{\boldsymbol{\Omega}}_{a}$, and $P_{0}, \omega_{a}, \hat{\boldsymbol{\Omega}}_{a}$, and $\phi$ are the pressure amplitude, angular frequency, propagation direction of the ultrasound $\left(\left|\hat{\boldsymbol{\Omega}}_{a}\right|=1\right)$, and local initial phase, respectively. With not very high ultrasound pressures, the optical index of refraction experiences a small perturbation approximated by $n(\mathbf{r}, t)=n_{0}\left[1+\eta P(\mathbf{r}, t) /\left(\rho v_{a}^{2}\right)\right]$, where $\rho$ is the fluid density; $v_{a}$ is the ultrasound speed; and $\eta$ is the elasto-optical coefficient. We obtain the increment $\delta=k_{0} n_{0}\left|\mathbf{r}_{b}-\mathbf{r}_{a}\right|+\varphi_{a, b}(t)$ of the optical phase along the free path between $\mathbf{r}_{a}$ and $\mathbf{r}_{b}$ by integrating the $k_{0} n(\mathbf{r}, t)$ along the path, where

$$
\varphi_{a, b}(t)=k_{0} n_{0}\left[\hat{\boldsymbol{\Omega}} \cdot\left[\mathbf{e}_{b}(t)-\mathbf{e}_{a}(t)\right]+\frac{\eta}{\rho v_{a}^{2}} \int_{\mathbf{r}_{a}}^{\mathbf{r}_{b}} P(\mathbf{r}, t) d r\right],
$$

and $k_{0}$ is the optical wave number in a vacuum. In Eq. (1), we approximate the distance between scatterers with $\left|\mathbf{r}_{b}-\mathbf{r}_{a}\right|+\hat{\boldsymbol{\Omega}} \cdot\left[\mathbf{e}_{b}(t)-\mathbf{e}_{a}(t)\right]$, where $\hat{\boldsymbol{\Omega}}\left|\mathbf{r}_{b}-\mathbf{r}_{a}\right|=\mathbf{r}_{b}-\mathbf{r}_{a}, \mathbf{e}_{s}(t)=\hat{\boldsymbol{\Omega}}_{a} P_{0} S_{a} /\left(k_{a} \rho v_{a}^{2}\right) \sin \left(\omega_{a} t-\mathbf{k}_{a} \cdot \mathbf{r}_{s}+\phi-\phi_{a}\right)$ is the ultrasound-induced displacement of the optical scatterer at $\mathbf{r}_{s}(s=a, b)$, and $S_{a}$ and $\phi_{a}$ are, respectively, deviations of the amplitude and the phase of the scatterer from the motion of the surrounding fluid. ${ }^{18}$ The second term on the right-hand side of Eq. (1) is the phase increment that is due to ultrasound-induced optical index of refraction changes.

We assume that the electrical field mutual coherence function $\Gamma\left(\mathbf{r}_{b^{\prime}}, t ; \mathbf{r}_{b^{\prime \prime}}, t+\tau\right)=\left\langle E\left(\mathbf{r}_{b^{\prime}}, t\right) E^{*}\left(\mathbf{r}_{b^{\prime \prime}}, t+\tau\right)\right\rangle$ for two closely spaced points $\mathbf{r}_{b^{\prime}}$ and $\mathbf{r}_{b^{\prime \prime}}$ is quasi-uniform, and we relate it to the time-varying specific intensity 
$I\left(\mathbf{r}_{b}, \hat{\mathbf{\Omega}}, t, \tau\right)$ by a spatial Fourier transform over the difference variable $\mathbf{r}_{b^{\prime}}-\mathbf{r}_{b^{\prime \prime}}$ in the center-of-gravity coordinate system, ${ }^{23-25}$ where $\mathbf{r}_{b}=\left(\mathbf{r}_{b^{\prime}}+\mathbf{r}_{b^{\prime \prime}}\right) / 2$, and \langle\rangle denotes the ensemble averaging. Let $E\left(\mathbf{r}_{a}, t\right)$ be the partial wave scattered at $\mathbf{r}_{a}$ toward $\mathbf{r}_{b}$. For isotropic scattering and $k_{a} l_{t r} \gg 1$, there is no correlation between $\varphi_{a, b}(t)$ and the other ultrasound-induced optical phase increments accumulated in $E\left(\mathbf{r}_{a}, t\right)$, and $I\left(\mathbf{r}_{b}, \hat{\mathbf{\Omega}}, t, \tau\right)$ is independent of time $t$. The phase term $\Delta \varphi=\varphi_{a, b}(t+\tau)-\varphi_{a, b}(t)$ is given by

$$
\begin{aligned}
\Delta \varphi=2 \Lambda \sin \left(\frac{1}{2} \omega_{a} \tau\right) \sin \left(\mathbf{k}_{a} \cdot \frac{\mathbf{r}_{b}-\mathbf{r}_{a}}{2}\right) \quad & \left\{S_{a} \hat{\mathbf{\Omega}} \cdot \hat{\mathbf{\Omega}}_{a} \sin \left(\omega_{a} t+\frac{1}{2} \omega_{a} \tau-\mathbf{k}_{a} \cdot \frac{\mathbf{r}_{a}+\mathbf{r}_{b}}{2}+\phi-\phi_{a}\right)\right. \\
& \left.-\frac{\eta}{\hat{\mathbf{\Omega}} \cdot \hat{\mathbf{\Omega}}_{a}} \sin \left(\omega_{a} t+\frac{1}{2} \omega_{a} \tau-\mathbf{k}_{a} \cdot \frac{\mathbf{r}_{a}+\mathbf{r}_{b}}{2}+\phi\right)\right\}
\end{aligned}
$$

where $\Lambda=2 k_{0} n_{0} P_{0} /\left(k_{a} \rho v_{a}^{2}\right)$. For $l_{t r} \approx 1 \mathrm{~mm}$ and $P_{0}<10^{5} \mathrm{~Pa}$, the phase term $\Delta \varphi$ satisfies $\Delta \varphi \ll 1$, and we approximate $\exp [i \Delta \varphi]$ with $1-\left|\mathbf{r}_{b}-\mathbf{r}_{a}\right| \mu_{t}\left\langle\Delta \varphi^{2}\right\rangle_{l_{t r}} / 2$. Here, $\mu_{t}=\mu_{s}+\mu_{a}$, and $\mu_{t}$, $\mu_{s}$, and $\mu_{a}$ are the optical extinction, scattering, and absorption coefficients, respectively. $\left\langle\Delta \varphi^{2}\right\rangle_{l_{t r}}$ is the average value of $\Delta \varphi^{2}$ in volume $V_{0} \sim l_{t r}^{3}$ per mean optical free path. The average of $\Delta \varphi^{2}$ over the center-of-gravity coordinate $\left(\mathbf{r}_{a}+\mathbf{r}_{b}\right) / 2$ in volume $V_{0}$ is given by

$$
\left\langle\Delta \varphi^{2}\right\rangle_{V_{0}}=2 \Lambda^{2} \sin ^{2}\left(\frac{1}{2} \omega_{a} \tau\right) \sin ^{2}\left(\mathbf{k}_{a} \cdot \frac{\mathbf{r}_{b}-\mathbf{r}_{a}}{2}\right)\left\{S_{a}^{2}\left(\hat{\boldsymbol{\Omega}} \cdot \hat{\boldsymbol{\Omega}}_{a}\right)^{2}+\frac{\eta^{2}}{\left(\hat{\boldsymbol{\Omega}} \cdot \hat{\boldsymbol{\Omega}}_{a}\right)^{2}}-2 S_{a} \eta \cos \left(\phi_{a}\right)\right\}
$$

and from the probability density of the free path $l$, which is $l_{t r}^{-1} \exp \left[-l / l_{t r}\right]$, we obtain

$$
\left\langle\Delta \varphi^{2}\right\rangle_{l_{t r}}=\Lambda^{2} \sin ^{2}\left(\frac{1}{2} \omega_{a} \tau\right) \frac{\left(l_{t r} \mathbf{k}_{a} \cdot \hat{\mathbf{\Omega}}\right)^{2}}{1+\left(l_{t r} \mathbf{k}_{a} \cdot \hat{\mathbf{\Omega}}\right)^{2}}\left[S_{a}^{2}\left(\hat{\mathbf{\Omega}} \cdot \hat{\mathbf{\Omega}}_{a}\right)^{2}+\frac{\eta^{2}}{\left(\hat{\mathbf{\Omega}} \cdot \hat{\mathbf{\Omega}}_{a}\right)^{2}}-2 \eta S_{a} \cos \left(\phi_{a}\right)\right] .
$$

The three terms in square brackets in Eq. (4) are related to the two mechanisms of modulation and the correlation between the phase increments produced by these mechanisms along the same free path, respectively. ${ }^{18}$ The increment of the intensity $I\left(\mathbf{r}_{b}, \hat{\mathbf{\Omega}}, \tau\right)$ that is due to the contribution of $I\left(\mathbf{r}_{a}, \hat{\mathbf{\Omega}}^{\prime}, \tau\right)$, which is scattered at $\mathbf{r}_{a}$ into direction $\hat{\boldsymbol{\Omega}}$, is equal to $\Delta I=I\left(\mathbf{r}_{a}, \hat{\mathbf{\Omega}}^{\prime}, \tau\right) \exp \left(-\mu_{t}\left|\mathbf{r}_{b}-\mathbf{r}_{a}\right|\right)\left[1-\left|\mathbf{r}_{b}-\mathbf{r}_{a}\right| \mu_{t}\left\langle\Delta \varphi^{2}\right\rangle_{l_{r}} / 2\right]$. By accumulating all of the increments along the $\hat{\boldsymbol{\Omega}}$ direction starting from some distant $\mathbf{r}_{0}$, we have

$$
I\left(\mathbf{r}_{b}, \hat{\mathbf{\Omega}}, \tau\right)=I_{0}\left(\mathbf{r}_{b}, \hat{\mathbf{\Omega}}, \tau\right)+\int_{\mathbf{r}_{0}}^{\mathbf{r}_{b}} \int_{4 \pi} \mu_{s} p\left(\hat{\mathbf{\Omega}}, \hat{\mathbf{\Omega}}^{\prime}\right) \Delta I d\left|\mathbf{r}_{b}-\mathbf{r}_{a}\right| d \hat{\mathbf{\Omega}}^{\prime}
$$

where $I_{0}\left(\mathbf{r}_{b}, \hat{\boldsymbol{\Omega}}, \tau\right)$ is due to the unscattered field. ${ }^{24}$ After applying $\hat{\boldsymbol{\Omega}} \cdot \nabla$ to Eq. (5), we obtain the CTE as

$$
\hat{\mathbf{\Omega}} \cdot \nabla I(\mathbf{r}, \hat{\mathbf{\Omega}}, \tau)=-\left(\mu_{a}+\mu_{s}\right) I(\mathbf{r}, \hat{\mathbf{\Omega}}, \tau)+S(\mathbf{r}, \hat{\mathbf{\Omega}})+\mu_{s} \int_{4 \pi} p\left(\hat{\mathbf{\Omega}}, \hat{\mathbf{\Omega}}^{\prime}\right)\left[1-\frac{1}{2}\left\langle\Delta \varphi^{2}\right\rangle_{l_{r}}\right] I\left(\mathbf{r}, \hat{\mathbf{\Omega}}^{\prime}, \tau\right) d \Omega^{\prime}
$$

In Eq. $(6), p\left(\hat{\mathbf{\Omega}}, \hat{\mathbf{\Omega}}^{\prime}\right)=1 /(4 \pi)$ is the isotropic scattering phase function, and $S(\mathbf{r}, \hat{\mathbf{\Omega}})$ is the monochromatic source term. Like in the case of Brownian motion, ${ }^{25}$ this equation can be obtained by pre-averaging the phase increments in a more rigorously derived CTE. ${ }^{21}$

To obtain the CDE, we apply the standard approximation $I(\mathbf{r}, \hat{\mathbf{\Omega}}, \tau) \approx[\Phi(\mathbf{r}, \tau)+3 \hat{\mathbf{\Omega}} \cdot \mathbf{J}(\mathbf{r}, \tau)] /(4 \pi)$ in Eq. $(6)$. $\Phi(\mathbf{r}, \tau)$ is actually the temporal field autocorrelation function related to the optical intensity spectrum by the temporal Fourier transform. The CDE is

$$
\nabla \cdot[D \nabla \Phi(\mathbf{r}, \tau)]-\left[\mu_{a}+\mu_{s} \hat{\varphi}(\tau)\right] \Phi(\mathbf{r}, \tau)+S_{0}(\mathbf{r})=0
$$

In Eq. $(7), D=\left(3 \mu_{s}\right)^{-1}$, and $\hat{\varphi}(\tau)$ is given by

$$
\hat{\varphi}(\tau)=\frac{1}{2} \Lambda^{2} \sin ^{2}\left(\frac{1}{2} \omega_{a} \tau\right)\left[\eta^{2}\left(k_{a} l_{t r}\right) \tan ^{-1}\left(k_{a} l_{t r}\right)+\frac{1}{3} S_{a}^{2}-2 \eta S_{a} \cos \left(\phi_{a}\right)\right] .
$$




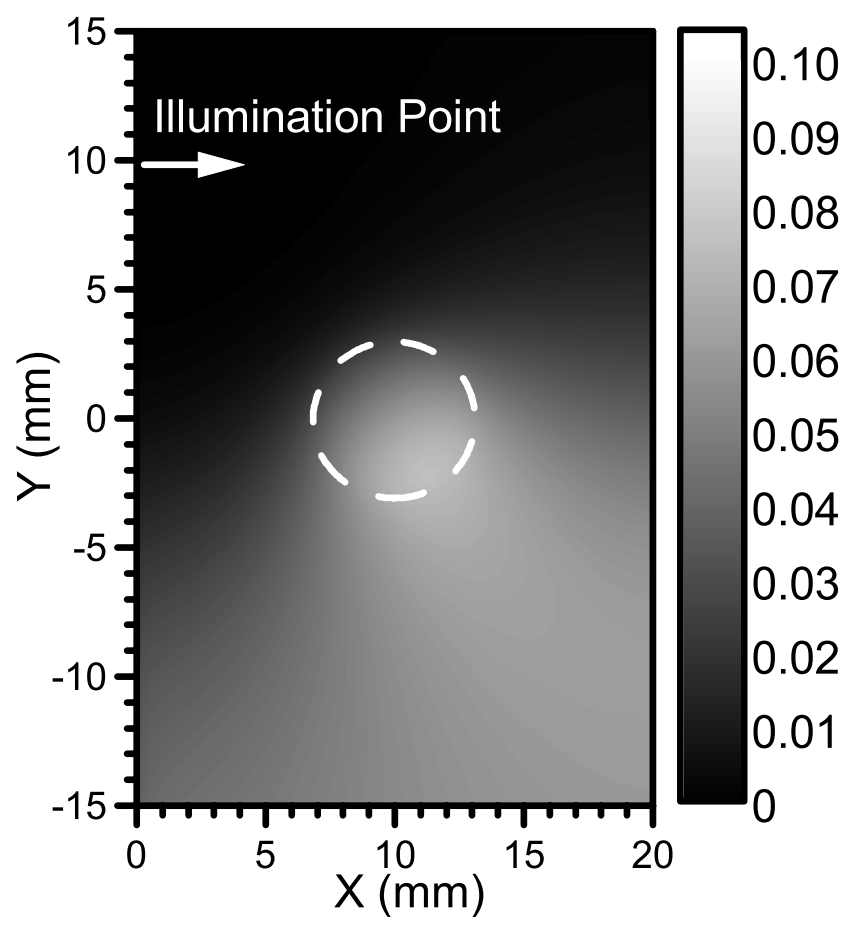

Figure 1. Modulation depth of the ultrasound-modulated light for an ultrasound cylinder in a scattering slab at $z=0$ mm. The white circle marks the ultrasound cross section.

\section{CYLINDER OF ULTRASOUND IN OPTICALLY SCATTERING SLAB}

In infinite media, the solution of Eq. (7) for monochromatic point source $S_{0}$ at the origin is

$$
\Phi(\mathbf{r}, \tau)=\frac{S_{0}}{4 \pi D} \frac{\exp \left(-r \sqrt{\frac{\mu_{a}+\mu_{s} \hat{\varphi}(\tau)}{D}}\right)}{r},
$$

which can be used to analytically study various configurations of ultrasound within scattering media. In finite media, the boundary conditions are identical to those in the diffusion equation used in radiative transfer. ${ }^{27}$ For matched optical properties, continuity requires that $\Phi(\mathbf{r}, \tau)$ and $D \partial \Phi(\mathbf{r}, \tau) / \partial n$ are constant across the boundary, where $\hat{\mathbf{n}}$ is an unity vector perpendicular to the boundary. For a scattering half space $(z>0)$ filled with ultrasound and irradiated by a pencil source from the free space, the boundary condition is $\Phi(\mathbf{r}, \tau)=2 D \partial \Phi(\mathbf{r}, \tau) / \partial z$, which leads to the extrapolated zero boundary position at $z=-2 D$.

We consider an infinitely wide scattering slab, with surface planes at $x=0 \mathrm{~mm}$ and $x=20 \mathrm{~mm}$. We assume $v_{a}=1480 \mathrm{~m} / \mathrm{s}, \rho=10^{3} \mathrm{~kg} / \mathrm{m}^{3}, \eta=0.32, \mu_{a}=0.1 \mathrm{~cm}^{-1}$; isotropic scattering with $l_{t r}=1 \mathrm{~mm}, n_{0}=1.33$ in whole space; $S_{a}=1$, and $\phi_{a}=0$, as typical values for soft biological tissues and for visible and near-infrared optical wavelengths. ${ }^{18}$ A cylinder of radius $a=3.175 \mathrm{~mm}$, infinitely long in the $Z$ direction, with an axis at $(x, y)=(10 \mathrm{~mm}, 0 \mathrm{~mm})$, is filled with a $5 \mathrm{MHz}$ continuous-wave ultrasound of pressure amplitude $P_{0}=10^{5} \mathrm{~Pa}$ traveling in the $Z$ direction. A pencil light source $S_{0}$ of wavelength $\lambda_{0}=532 \mathrm{~nm}$ irradiates the slab along the $X$ direction at $(x, y, z)=(0 \mathrm{~mm}, 10 \mathrm{~mm}, 0 \mathrm{~mm})$. Eq. $(7)$ has solutions $\Phi_{\text {inc }}\left(\mathbf{r}_{d}, \tau\right), \Phi_{s c}\left(\mathbf{r}_{d}, \tau\right)$, and $\Phi_{\text {in }}\left(\mathbf{r}_{d}, \tau\right)$, which are for the autocorrelation functions incident from the source, scattered from the cylinder, and inside the cylinder, respectively. ${ }^{29}$ If the cylinder axis is at the origin, then we have

$$
\Phi_{\{. .\}}\left(\mathbf{r}_{d}, \tau\right)=\sum_{n=0}^{+\infty} \cos \left(n \phi_{d}\right) \int_{0}^{+\infty} \cos \left(p z_{d}\right) \Psi_{\{. .\}}(p) d p
$$


where $\Psi_{i n c}(p)=H_{n}\left(x_{>}\right) I_{n}\left(x_{<}\right), \Psi_{s c}(p)=B_{n}(p) K_{n}(x)$, and $\Psi_{i n}(p)=C_{n}(p) I_{n}(y) ; \mathbf{r}_{s}=\left(\rho_{s}, \phi_{s}, z_{s}\right)$ and $\mathbf{r}_{d}=$ $\left(\rho_{d}, \phi_{d}, z_{d}\right)$ are positions of the point source and the detector in the cylindrical coordinates, and $I_{n}$ and $K_{n}$ are modified Bessel functions of the first and second kind, respectively; $x_{\lessgtr}=\rho_{\lessgtr} \sqrt{p^{2}-k_{\text {out }}^{2}} ; \rho_{\lessgtr}=\min (\max )\left[\rho_{s}, \rho_{d}\right]$; $x=\rho_{d} \sqrt{p^{2}-k_{\text {out }}^{2}} ; y=\rho_{d} \sqrt{p^{2}-k_{\text {in }}^{2}} ; k_{\text {in }}^{2}=-\left[\mu_{a}+\mu_{s} \hat{\varphi}(\tau)\right] / D ; k_{\text {out }}^{2}=-\mu_{a} / D ; B_{n}$ and $C_{n}$ are given by

$$
\begin{aligned}
B_{n}(p) & =-H_{n}\left(z_{b}\right) \frac{x_{b} I_{n}^{\prime}\left(x_{b}\right) I_{n}\left(y_{b}\right)-y_{b} I_{n}^{\prime}\left(y_{b}\right) I_{n}\left(x_{b}\right)}{x_{b} K_{n}^{\prime}\left(x_{b}\right) I_{n}\left(y_{b}\right)-y_{b} I_{n}^{\prime}\left(y_{b}\right) K_{n}\left(x_{b}\right)}, \\
C_{n}(p) & =-H_{n}\left(z_{b}\right) \frac{x_{b} I_{n}^{\prime}\left(x_{b}\right) K_{n}\left(x_{b}\right)-x_{b} K_{n}^{\prime}\left(x_{b}\right) I_{n}\left(x_{b}\right)}{x_{b} K_{n}^{\prime}\left(x_{b}\right) I_{n}\left(y_{b}\right)-y_{b} I_{n}^{\prime}\left(y_{b}\right) K_{n}\left(x_{b}\right)},
\end{aligned}
$$

where $H_{n}\left(z_{b}\right)=\left[(\operatorname{sgn}(n)+1) S_{0} K_{n}\left(z_{b}\right)\right] /\left(2 \pi^{2} D\right), x_{b}=a \sqrt{p^{2}-k_{\text {out }}^{2}}, y_{b}=a \sqrt{p^{2}-k_{\text {in }}^{2}}, z_{b}=\rho_{s} \sqrt{p^{2}-k_{\text {out }}^{2}}$, and $\operatorname{sgn}(n)$ is the sign function. We use Eq. (10) to obtain values for the modulation depth, defined as the amplitude ratio of the first to the zero harmonic of the modulated light. Three pairs of independent cylinder images ${ }^{28}$ are used to satisfy the boundary conditions. The modulation depth is calculated in the whole volume of the scattering slab, and Fig. 1 presents its values in the $z=0 \mathrm{~mm}$ plane. The results show that the modulation depth is higher away from the source, in the shadow of the cylinder, due to the counteracting contributions of the modulated and unmodulated light.

\section{CONCLUSION}

In conclusion, we derived the CTE and the CDE for ultrasound-modulated light, which are valid for optical and ultrasound spatial inhomogeneities on the order of $l_{t r}$, for moderate ultrasound pressures and frequencies satisfying $k_{a} l_{t r} \gg 1$. The CDE is of use for the estimation of sensitivity and signal-to-noise ratios in UOT, where both heterogeneous ultrasound fields and optical parameters are encountered. It can be solved analytically or numerically by the many methods developed for the diffusion equation. Based on the CDE, we obtained an analytical solution for the modulation depth of ultrasound-modulated light produced by a cylinder of ultrasound placed in an optically scattering slab. More challenging setups, with highly focused ultrasound and very high ultrasound pressure, should be the subject of further theoretical investigations.

This project was sponsored by NIH grant R33 CA 094267.

\section{REFERENCES}

1. F. A. Marks, H. W. Tomlinson, and G. W. Brooksby, "A comprehensive approach to brest cancer detection using light: photon localization by ultrasound modulation and tissue characterization by spectral discrimination," in Proc. SPIE, 1888, pp. 500-510, 1993.

2. L. V. Wang, S. L. Jacques, and X. Zhao, "Continuous-wave ultrasonic modulation of scattered laser light to image objects in turbid media," Opt. Lett. 20, pp. 629-631, 1995.

3. L. V. Wang and G. Ku, "Frequency-swept ultrasound-modulated optical tomography of scattering media," Opt. Lett. 23, pp. 975-977, 1998.

4. S. Leveque, A. C. Boccara, M. Lebec, and H. Saint-Jalmes, "Ultrasonic tagging of photon paths in scattering media: parallel speckle modulation processing," Opt. Lett. 24, pp. 181-183, 1999.

5. G. Yao, S.-L. Jiao, and L. V. Wang, "Frequency-swept ultrasound-modulated optical tomography in biological tissue by use of parallel detection," Opt. Lett. 25, pp. 734-736, 2000.

6. A. Lev, Z. Kotler, and B. G. Sfez, "Ultrasound tagged light imaging in turbid media in a reflectance geometry," Opt. Lett. 25, pp. 378-380, 2000.

7. J. Li, G. Ku, and L. V. Wang, "Ultrasound-modulated optical tomography of biological tissue by use of contrast of laser speckles," Appl. Optics 41, pp. 6030-6035, 2002.

8. M. Gross, P. Goy, and M. Al-Koussa, "Shot-noise detection of ultrasound-tagged photons in ultrasoundmodulated optical imaging," Opt. Lett. 28, pp. 2482-2484, 2003.

9. T. W. Murray, L. Sui, G. Maguluri, R. A. Roy, A. Nieva, F. Blonigen, and C. A. DiMarzio, "Detection of ultrasound-modulated photons in diffuse media using the photorefractive effect," Opt. Lett 29, pp. 25092511, Nov 2004. 
10. S. Sakadzic and L. V. Wang, "High-resolution ultrasound-modulated optical tomography in biological tissues," Opt. Lett 29, pp. 2770-2772, Dec 2004.

11. G. Maret and P. E. Wolf, "Multiple light-scattering from disordered media - the effect of brownian-motion of scatterers," Z. Phys. B 65, pp. 409-413, 1987.

12. W. Leutz and G. Maret, "Ultrasonic modulation of multiply scattered-light," Physica B 204, pp. 14-19, 1995.

13. M. Kempe, M. Larionov, D. Zaslavsky, and A. Z. Genack, "Acousto-optic tomography with multiply scattered light," J. Opt. Soc. Am. A 14, pp. 1151-1158, 1997.

14. G. D. Mahan, W. E. Engler, J. J. Tiemann, and E. G. Uzgiris, "Ultrasonic tagging of light: Theory," in Proc. Natl. Acad. Sci. USA, 95, p. 14015, 1998.

15. L. V. Wang, "Mechanisms of ultrasonic modulation of multiply scattered coherent light: an analytic model," Phys. Rev. Lett. 87, pp. 043903-(1-4), 2001.

16. S. Sakadzic and L. V. Wang, "Ultrasonic modulation of multiply scattered coherent light: an analytical model for anisotropically scattering media," Phys. Rev. E 66, pp. 026603-(19), 2002.

17. A. Lev and B. Sfez, "In vivo demonstration of the ultrasound modulated light technique," J. Opt. Soc. Am. A 20, pp. 2347-2354, 2003.

18. S. Sakadzic and L. V. Wang, "Modulation of multiply scattered coherent light by ultrasonic pulses: An analytical model," Phys. Rev. E 72, pp. 036620-(1-12), 2005.

19. L. V. Wang, "Mechanisms of ultrasonic modulation of multiply scattered coherent light: a monte carlo model," Opt. Lett. 26, pp. 1191-1193, 2001.

20. D. J. Pine, D. A. Weitz, P. M. Chaikin, and E. Herbolzheimer, "Diffusing-wave spectroscopy," Phys. Rev. Lett. 60, pp. 1134-1137, 1988.

21. S. Sakadzic and L. V. Wang, "Correlation transfer equation for ultrasound-modulated multiply scattered light." in preparation.

22. U. Frisch, Wave propagation in random media, vol. 1 of Probabilistic methods in applied mathematics, pp. 75-198. Academic, New York, 1968.

23. Y. Barabanenkov, A. Vinogradov, Y. Kravtsov, and V. Tatarskii, "Application of the theory of multiple scattering of waves to the derivation of the radiation transfer equation for a statistically inhomogeneous medium," Radiophys. Quantum Electron. 15, pp. 1420-1425, 1972.

24. A. Ishimaru, Wave Propagation and Scattering in Random Media, Academic, New York, 1978.

25. R. L. Dougherty, B. J. Ackerson, N. M. Reguigui, F. Dorri-Nowkoorani, and U. Nobbmann, "Correlation transfer: development and application," J. Quant. Spectrosc. Radiat. Transfer 52, pp. 713-727, 1994.

26. D. A. Boas, L. E. Campbell, and A. G. Yodh, "Scattering and imaging with diffusing temporal field correlations," Phys. Rev. Lett. 75, pp. 1855-1858, 1995.

27. R. C. Haskell, L. O. Svaasand, T.-T. Tsay, T.-C. Feng, M. S. McAdams, and B. J. Tromberg, "Boundary conditions for the diffusion equation in radiative transfer," J. Opt. Soc. Am. A 11, pp. 2727-2741, 1994.

28. M. S. Patterson, B. Chance, and B. C. Willson, "Time resolved reflectance and transmittance for the noninvasive measurement of tissue optical-properties," Appl. Opt. 28, pp. 2331-2336, 1989.

29. J. D. Jackson, Classical electrodynamics, Wiley, New York, 3 ed., 1998.

30. J. Jensen and N. B. Svendsen, "Calculation of pressure fields from arbitrarily shaped, apodized, and excited ultrasound transducers," IEEE Trans. Ultrason., Ferroelec., Freq. Contr. 39, pp. 262-267, 1992. 